\title{
Исследование эффекта электронного охлаждения. Обзор современного состояния работ
}

\author{
() Н.О. Безверхний, ${ }^{1,2}$ С.В. Бобашев, ${ }^{1,2}$ А.В. Колычев, ${ }^{3}$ Н.А. Монахов, ${ }^{1,9}$ С.А. Поняев, ${ }^{1}$ В.А. Сахаров ${ }^{1}$ \\ ${ }^{1}$ Физико-технический институт им. А.Ф. Иоффре РАН, \\ 194021 Санкт-Петербург, Россия \\ ${ }^{2}$ Санкт-Петербургский политехнический университет Петра Великого, \\ 195251 Санкт-Петербург, Россия \\ ${ }^{3}$ Балтийский государственный технический университет „Военмех“ им. Д.Ф. Устинова, \\ 190005 Санкт-Петербург, Россия \\ ฯ e-mail: nm1988@mail.ru
}

(Поступило в Редакцию 12 сентября 2018 г.)

Рассмотрены результаты исследований эффекта электронного охлаждения. Проведен анализ современного состояния исследований. Обсуждены возможности экспериментального изучения явления электронного охлаждения на базе большой ударной трубы и плазмогазодинамической установки ФТИ им. А.Ф. Иоффе.

DOI: 10.21883/JTF.2019.03.47162.340-18

\section{Введение}

Тепловая защита летательного аппарата, движущегося с гиперзвуковой скоростью в атмосфере Земли, остается одной из основных проблем космической и авиационной отраслей [1]. Используемые в настоящее время пассивные методы теплозащиты связаны с заметным увеличением веса летательного аппарата и, как следствие, ограничением массы полезного груза, выводимого на орбиту. В последние десятилетия рассматриваются альтернативные методы тепловой защиты, основанные на использовании электрического и магнитного полей с целью воздействия на поток ионизованного газа в окрестности летательного аппарата [2-5]. Однако на значительной части траектории спуска возвращаемого космического аппарата и гиперзвукового летательного аппарата нагрев осуществляется преимущественно за счет излучения. В таких условиях необходимо осуществлять теплоотвод более интенсивный, чем могут позволить пассивные методы теплозащиты. В качестве одного из методов активной теплозащиты рассматривается электронное охлаждение (ЭО). В основе технологии ЭО лежит явление термоэлектронной эмиссии, сопровождающееся охлаждением эмиттирующей поверхности за счет уноса энергии электронами.

Практическая реализация метода ЭО применительно к летательным аппаратам является достаточно сложной задачей. В настоящий момент по данной тематике нам известны только теоретические работы, связанные с численными оценками и математическим моделированием. Первые работы, в которых был описан способ генерации электрического тока при помощи термоэмиссионных материалов на поверхности гиперзвукового летательного аппарата, появились еще в 1960-х годах [6,7]. В этих работах проводится анализ и экспериментальное исследование новой конструкции термоэлектронного генератора электрического тока для летательных аппаратов. Принцип такого генератора заключается в использовании термоэмиссионных материалов на поверхности головно- го обтекателя для генерации электрического тока под воздействием высоких температур вследствие аэродинамического нагрева. Авторами [6] получены уравнения для оценки получаемого на выходе генератора тока, выходной мощности и условий максимальной мощности. Чтобы смоделировать условия полета, тестовая модель помещалась в плазменную струю азота или аргона. В качестве материала для эмиттера и коллектора были выбраны графит, торированный вольфрам, вольфрам и молибден. Из выбранных материалов, однако, только графит в некоторой степени соответствовал требованиям, необходимым для работы гиперзвукового плазменного термоэлектронного генератора. В этих опытах было обнаружено, что ток эмиссии графита в присутствии высокотемпературного газового потока намного выше значений, приведенных в справочной литературе.

В работах $[6,7]$ не обсуждается возможность применения термоэмиссионных материалов для активной теплозащиты ЭО. На тот момент достижение летательными аппаратами необходимых скоростей было невозможным, поэтому технология не получила развития. Первые инженерные предложения о возможности применения ЭО в системах теплозащиты элементов высокоскоростных летательных аппаратов (ВЛА), по-видимому, принадлежат отечественным специалистам [8] и датируются 2009 годом. В статьях и патентах [8-18] предлагаются конструктивные решения, направленные на обеспечение ЭО передних кромок крыльев и носовых частей ВЛА, разработаны инженерные методики и проведены оценки теплового эффекта ЭО. Начиная с 2014 года, публикуются проводимые в США активные исследования по численному моделированию эффекта ЭО. Проводятся расчеты эффективности переноса энергии электронами при различных внешних условиях с учетом состав среды, скорости набегающего потока, различных геометрических параметрах исследуемых моделей. В работах [19-23] показано, что путем оптимизации параметров модели и скорости набегающего потока можно добиться на снижения температуры поверхности до 40\%. 


\section{Обзор работ}

В 2003 году в США Управлением перспективных исследовательских проектов (DARPA) и BBC CША была инициирована совместная программа под названием Falcon, предназначенная для развития технологий, необходимых для обеспечения устойчивости гиперзвукового полета [24]. В рамках программы в 2010 и 2011 годах было произведено несколько гиперзвуковых летных испытаний устройств, которые получили название Hypersonic Technology Vehicle 2. Для увеличения максимальной дальности полета и аэродинамического качества гиперзвуковые летательные аппараты должны обладать заостренной геометрией передних кромок крыла и носовой части. Однако острые края подвержены сильному нагреву. Так, теоретический анализ, выполненный в [25], показывает, что в точке стагнации коэффициент теплопроводности обратно пропорционален радиусу кривизны профиля переднего края летательного аппарата. Интенсивный аэродинамический нагрев приводит к тому, что при гиперзвуковых скоростях полета температура на поверхности головной части летательного аппарата может превышать $2000 \mathrm{~K}$. Для изоляции деталей от экстремальных температур на гиперзвуковых летательных аппаратах используют системы тепловой защиты с применением жаростойких, тугоплавких материалов. Однако их многократное использование невозможно по причине частичного или полного разрушения под действием высоких температур [26].

В работе [19] отмечается, что одним из подходов к решению проблемы высоких локальных скоростей нагрева является использование сверхвысокотемпературных композитных материалов (UHTC) на передних частях конструкции гиперзвуковых летательных аппаратов. Этот подход был применен на экспериментальном гиперзвуковом самолете НАCA X-43 [27]. Однако UHTC имеют такие ограничения, как хрупкость и относительно большой вес, что сужает рамки их применимости [28]. Некоторых проблем можно избежать при использовании термоэлектрических материалов, которые генерируют электрический ток под воздействием высоких температур, что может привести к эффекту электронного охлаждения поверхности. Предполагая, что материалы с такими термоэлектрическими и физическими характеристиками существуют, авторы проводят анализ условий среды вблизи поверхности летательного аппарата с использованием вычислительной гидродинамики.

Представлены результаты анализа возможности использования эффекта ЭО. При помощи кода CFD LeMANS, предназначенного для моделирования термоэлектронной эмиссии с поверхности летательного аппарата, описана реализация граничного условия с учетом эмиссии электронов. Авторами проведено исследование с целью определения влияния на эффект охлаждения величины работы выхода материала, скорости свободного потока и геометрии обтекателя на эффект охлаждения. Численные расчеты показывают, что при уменьшении работы выхода материала, увеличении скорости набегающего потока и уменьшении радиуса конструкции головного обтекателя эффект охлаждения может возрастать в силу увеличения тока эмиссии. Продемонстрировано эффективное уменьшение работы выхода электронов с поверхности под действием приложенного электрического поля, возникающего в ударном слое. Численные результаты также показывают, что электрическое поле, создаваемое наведенным зарядом эмитированных электронов, не оказывает существенного влияния на предсказанные свойства. При расчетах предполагались следующие условия полета: набегающий поток с числом Маха $M=19.4$ на высоте $60 \mathrm{~km}$ обтекает профиль с радиусом носовой части $R=1.0 \mathrm{~cm}$. Результаты моделирования показали, что термоэмиссионное излучение может снизить температуру поверхности примерно на 40\% при условии, что работа выхода материала составляет $2.0 \mathrm{eV}$. Полученные результаты показали многообещающие перспективы использования термоэмиссионных материалов и их охлаждающих свойств. Однако осталась необходимость расширения исследований, учет в модели различия условий на разных высотах, а также обтекания под различными углами атаки. При расчете не была учтена рекомбинация электронов на поверхность и ряд других параметров.

В работе [20] результаты математического моделирования эффекта ЭО высокоэнтропийного слоя сравниваются с результатами экспериментальных исследований по генерации тока с использованием термоэмиссионных материалов, проводимых в аэродинамической трубе с электродуговым подогревателем в плазме азота и аргона $[6,7]$. Сравнения проводятся для высокоэнтропийных слоев азота и аргона: 7000 и $3000 \mathrm{kcal} / \mathrm{kg}$ соответственно, с числом Маха $M=(2.5-3)$. Обсуждается преобразование приведенных энтальпий и чисел Маха в температуры и скорости набегающего потока. Описан метод численного моделирования с помощью кода CFD LeMANS, включающий описание граничного условия и учитывающий электронную эмиссию с поверхности. Описана применяемая химическая модель ионизации аргона. В качестве материалов был выбран графит и вольфрам. Для сравнения используются две различные геометрии обтекателя с различным радиусом кривизны. В работе проводится сравнение современных подходов к моделированию термоэлектронной эмиссии с экспериментами [6,7] в которых варьировали параметры набегающего потока, эмиссионные свойства материалов и геометрия обтекателя. Использовались две различные геометрии обтекаемой потоком модели. Одна из них представляет собой осесимметричный конус с закругленной носовой частью радиусом $0.73 \mathrm{~cm}$, за которым следуют сегмент с углом раствора $10^{\circ}$, небольшой цилиндрический сегмент, затем конусообразный с углом раствора $6^{\circ}$. Эмиттирующая область выполнена из графита имеет площадь $8.4 \mathrm{~cm}^{2}$. Другая модель представляет собой осесимметричный конус с закругленной носовой частью радиусом $1.0 \mathrm{~cm}$ с углом раствора $13.5^{\circ}$ с эмитирующей поверхностью площадью $16 \mathrm{~cm}^{2}$. 
Исследование показало, что выбор модели с радиусом носовой части $0.73 \mathrm{~cm}$, работы выхода $4.8 \mathrm{eV}$ и аргона в качестве среды, приводят к повышению температуры поверхности. Однако эта тенденция не наблюдается для геометрии с радиусом носовой части $1.0 \mathrm{~cm}$, работой выхода $4.48 \mathrm{eV}$ и азота в качестве среды, где наблюдается снижение температуры поверхности и увеличение конвективного теплообмена. Таким образом, показано, что электронная эмиссия может как понижать, так и повышать температуру поверхности в зависимости от интенсивности эмиссии, работы выхода материала, условий среды и характеристик набегающего потока. Результаты моделирования показали, что при обтекании модели аргоном температура поверхности выше, чем в аналогичных условиях с обтеканием азотом. Численные результаты дают широкий диапазон значений эмиссионного тока из-за неопределенности в характеристиках набегающего потока и эмиссионных свойств материала, но при этом хорошо согласуются с экспериментами. Результаты, как правило, выше экспериментальных для азота и ниже для аргона. Это происходит из-за того, что численная модель не учитывает рекомбинацию электронов обратно на поверхность. Существует также некоторая неопределенность в экспериментальных измерениях параметров течения и тока. В данной модели предполагается, что работа выхода материала поверхности постоянна, в то время как она может изменяться по мере деградации материала с течением времени под воздействием высоких температур. Результаты также демонстрируют, что эффект ЭО поверхности наступает при определенном минимальном значении эмиссии. Хотя результаты моделирования хорошо согласуются с экспериментами по генерации тока [6], авторы заявляют о необходимость дальнейшей работы по улучшению физической точности и возможностей численного моделирования. При расчете не было учтено влияние диффузии заряженных частиц под действием электрического поля. Авторами разработаны рекомендации по улучшению физической точности математического моделирования.

В работе [29] также предложена методика ЭО для отвода тепла от сложных для охлаждения мест, таких, как гиперзвуковые передние кромки или лопасти турбины реактивного двигателя. Подробно обсуждается эффективность ЭО относительно других методов охлаждения. Так, авторы указывают на значительные недостатки метода воздушного охлаждения применительно к узким кромкам ведущих частей летательного аппарата. В этой работе рассматривается физика эффекта, ее приложения и некоторые общие системные соображения. Дается краткое обсуждение применения ЭО для охлаждения лопастей турбин реактивного двигателя. Обсуждаются пределы применимости с точки зрения параметров полета. Представлена одномерная модель для расчета температуры поверхности летательного аппарата с учетом ЭО. Результаты показывают, что применение ЭО может снизить температуру гиперзвуковых летательных аппаратов на величину порядка $1000 \mathrm{~K}$. В заключении даны некоторые сравнения с существующими работами по ЭО и обсуждаются дополнительные физические процессы, необходимые для повышения точности модельных расчетов.

В работе [21] обсуждаются две аналитические модели ограничения пространственного заряда, который образуется вблизи поверхности и может влиять на эмиссию электронов. Первая модель предполагает, что электроны испускаются с поверхности холодными, а вторая, что испускаемые электроны имеют конечную температуру. Теория показывает, что эмиссия электронов, имеющих конечную температуру (в терминологии авторов работы „теплая эмиссия“), может приводить к большим значениям плотности тока, от которого напрямую зависит охлаждающий эффект. Модель ограничения пространственного заряда реализована с использованием одномерной кинетической модели плазмы. Плазма считается бесстолкновительной. Результаты моделирования хорошо согласуются с теорией, изложенной в [30]. Обе модели реализованы с помощью кода CFD LeMANS c тестовыми параметрами среды и геометрии обтекателя, описанными выше для предыдущих работ. Результаты показывают, что модель эмиссии электронов с конечной температурой приводит к большему снижению температуры поверхности. Эта тенденция имеет смысл, поскольку электроны, испускаемые с конечной температурой (теплая эмиссия), имеют большие энергии, что позволит им легче преодолевать потенциальный барьер виртуального катода. Эмиссия электронов с более высокой температурой приводит к более высокому ограничению пространственного заряда. Это свидетельствует о важности температуры излучаемых электронов.

В работе [31] исследовалась эмиссия с поверхности, которая представлялась покрытой электронами или как отрицательно заряженная поверхность. Было показано, что для эффективного охлаждения поверхность должна быть заряжена отрицательно. Это возможно, если собрать электроны эмиссии ниже по течению и замкнуть цепь на эмитирующей поверхности. Электроны испускаются с поверхности летательного аппарата (катода) и собираются ниже по течению (анод), подобно тому, как это происходит в двойном эмиссионном зонде. Эмиссионные зонды широко изучены и используются в экспериментальной физике плазмы [32-34]. Предполагалось, что потенциал поверхности может быть смещен относительно квазинейтрального потока до желаемого уровня, тогда как реальное значение потенциала будет зависеть от внешних условий.

Важным ограничением в модели эмиссионного зонда является образование объемного заряда, ограничивающего эмиссию. Для определения влияния объемного заряда на эмиссию электронов с поверхности авторами был использован оригинальный метод численного решения кинетических уравнений, разработанный в Университете Мичигана [35]. Используемый метод представляет собой альтернативный подход к решению задач с участием многих частиц и является более детерминированным. В нем используется дискретное фазовое пространство, в котором кинетические уравнения 
решаются напрямую. В расчете не было учтено влияние столкновений в плазме. В последующих работах авторы планируют провести моделирование ограничивающего действия пространственного заряда на эмиссию электронов для широкого диапазона значений потенциала поверхности, а также исследовать влияние эффекта ЭО на весь летательный аппарат с учетом сбора электронов эмиссии ниже по течению. Авторы подчеркивают необходимость экспериментального исследования эффекта для проверки результатов численного моделирования.

В работе [23] представлены результаты моделирования эффекта ЭО головного обтекателя гиперзвукового летательного аппарата с использованием вычислительной гидродинамики и программы моделирования, с помощью которой исследуется внутренняя теплопроводность материала. Цель работы заключается в оценке влияния внутренней теплопроводности на эффект ЭО. Авторами развит подход к моделированию для эффекта ЭО, который включает граничные условия, учитывающие термоэлектронную эмиссию с поверхности, электрическое поле вблизи поверхности, ограничение пространственного заряда. Для определения тепловых потоков вглубь поверхности был использован разработанный в университете Мичигана код MOPAR, позволяющий решать многомерные задачи, в том числе для материалов с температурной зависимостью и анизотропией параметров. Было выполнено исследование для ряда тепловых свойств термоэмиссионных материалов, а также получены глубинные профили проводимости. Результаты моделирования показывают, что учет внутренней теплопроводности материала приводит к большему снижению температуры поверхности. Авторы связывают это с тем, что внутренняя теплопроводность обеспечивает переход энергии через поверхность вглубь материала. Однако даже при учете внутренней теплопроводности, эффект ЭО остается основным фактором уменьшения температуры поверхности.

Авторы отмечают необходимость полномасштабного моделирования летательного аппарата с применением технологии ЭО. С его помощью можно будет проследить за электронами эмиссии, попавшими в поток, что позволит оценить их влияние на заряд конструкций аппарата. В последующих работах планируется более точно оценить влияние проводимости в глубине поверхности на обсуждаемые эффекты.

В работе [36] дается более полная теория физических процессов, существенных для понимания эффекта ЭО. Цель этой работы состояла в исследовании влияния физических процессов в плазменном слое на эффективность ЭО. Рассмотрены две различные аналитические модели ограничения эмиссии объемным зарядом. В первой модели предполагается, что электроны испускались с поверхности холодными. Во второй модели предполагается, что испускаемые электроны имеют конечную температуру. Теория показала, что электроны, испускаемые с конечной электронной температурой, обеспечивают более высокие значения эмиссии до момента образования виртуального катода, который ограничивает ток эмиссии. Теория теплой эмиссии была расширена, чтобы включить сверхзвуковые скорости ионов на границе оболочки. Моделирование хорошо согласуется с теорией теплой эмиссии, представленной в [30]. Также был представлен подход для аппроксимации электронной температуры. Результаты моделирования показали, что при более низких скоростях свободного потока эмиссия ограничена объемным зарядом, что уменьшает преимущества ЭО. Однако при увеличении скорости набегающего потока представленный подход позволяет получить снижение температуры поверхности до $50 \%$.

В России в 2011-2013 годах были зарегистрированы патенты по электронному охлаждению элементов газотурбинных установок и авиационных газотурбинных двигателей, а именно патенты № 2573551 „Способ охлаждения лопаток турбин газотурбинных установок“ [37], № 2578387 “Устройство охлаждения лопаток турбин газотурбинных установок“ [38] и патент на полезную модель № 151082 [39].

\section{Заключение}

Рассмотренные выше работы демонстрируют высокий интерес к разработке новых методических подходов к проблеме теплозащиты летательных аппаратов на примере метода ЭО. Анализируя содержание данных работ, можно заключить, что фундаментальных физических ограничений для применения метода ЭО нет. Одной из главных на сегодняшний день проблем, стоящих на пути практической реализации данного метода, является отсутствие материалов с необходимыми физическими свойствами, однако в связи с широкой применимостью термоэмиссионных преобразователей поиск новых материалов является активно развиваемым направлением. Также активно ведется поиск новых способов уменьшения работы выхода, о чем говорит высокая публикационная активность [40-43]. Все вышесказанное свидетельствует о приоритете РФ перед иностранными корпорациями и научными учреждениями в области исследований, направленных на обеспечение электронного охлаждения элементов ВЛА. Однако расчетные исследования по данному направлению проводятся и в США, что подтверждается большим количеством регулярно появляющихся в печати публикаций. Поэтому не вызывает сомнений необходимость проведения в научных организациях РФ комплексных научно-исследовательских и опытно-конструкторских работ по представленной в настоящей работе тематике.

Существенным, на наш взгляд, является проведение экспериментальных исследований различных физических аспектов ЭО. Так, в рамках проекта РФФИ 18-3800346 мол_а на базе ФТИ им. А.Ф. Иоффе в настоящий момент проводятся исследования, направленные на экспериментальное обнаружение изменения температуры термоэмиссионных материалов в условиях, приближенных к гиперзвуковому полету. Экспериментальные 
исследования выполняются на плазмогазодинамической установке, позволяющей создавать не ограниченный стенками газовый разряд в различных газовых средах (инертные газы, азот, воздух) при различных давлениях. Установка позволяет использовать в качестве газовой среды различные инертные газы, а также создавать газовый разряд в воздухе. Данная установка позволяет использовать энергию газового разряда как источник интенсивного нагрева и обойтись в эксперименте без применения гиперзвуковых ударных труб. К тому же образующуюся вблизи поверхности гиперзвукового летательного аппарата ионизированную среду моделирует плазма газового разряда. Следует заметить, что возможность регулировать давление в рабочей камере установки позволяет создать условия, близкие к реализуемым на высоте гиперзвуковых полетов $h \sim 80 \mathrm{~km}$. В рамках проекта будет произведена модернизация стенда для исследования химической стойкости термоэмиссионных материалов в условиях агрессивной среды.

В Физико-техническом институте им. А.Ф. Иоффе создан Газодинамический Испытательный Стенд (ГИС ФТИ), который представляет собой двухдиафрагменную ударную трубу, рассчитанную на получение и исследование ударных волн большой интенсивности. Реализация в ГИС ФТИ режимов течения со сшитой контактной поверхностью обеспечивает получение высоких значений параметров торможения рабочего газа при значениях числа Маха ударной волны вплоть до $M=15$ [44]. Постоянство параметров в области ударно нагретого газа сохраняется при этом в течение времени более $1 \mathrm{~ms}$. В комплексе ударная труба-сопло возможно проведение исследований взаимодействия струй различных газов между собой с преградой в поле течения, обтекания тел потоком различных газов, а также изучение физических явлений, имеющих место в сверхзвуковом расширяющемся потоке. Перечисленные выше особенности, а также многолетний опыт эксплуатации установки дают основания утверждать, что ГИС ФТИ является прецизионным инструментом в газодинамических исследованиях, позволяющим реализовать в эксперименте уникальные по параметрам газоплазменные состояния.

Работа выполнена при поддержке РФФИ в рамках проекта 18-38-00346 мол_а.

\section{Список литературы}

[1] Leyva I.A. // Physics Today. 2017. Vol. 70. N 11. P. 31-36.

[2] Лапушкина Т.А., Ерофеев А.В., Поняев С.А. // ЖТФ. 2011. Т. 81. Вып. 5. С. 28-34.

[3] Бобамев С.В., Менде Н.П., Попов П.А., Сахаров В.А. // ЖТФ. 2010. Т. 80. Вып. 12. С. 51-56.

[4] Бобамев С.В., Головачев Ю.П., Курбатов Г.А., Менде Н.П., Сахаров В.А., Чернышев А.С., Шмидт А.А. // ЖТФ. 2009. Т. 79. Вып. 1. С. 36-44.

[5] Сахаров В.А., Менде Н.П., Бобамев C.B., Van Wie D.M. // Письма в ЖТФ. 2006. Т. 32. Вып. 14. С. 40-46.

[6] Touryan K.J. // AIAA Journal. 1965. Vol. 3. N. 4. P. 652-659.
[7] LeBlank A.R., Grannemann W.W. // Proceedings of the IEEE. 1964. P. $1302-1310$.

[8] Кольчев А.В., Керножицкий В.А. // Решетневские чтения. 2009. T. 1. № 13. C. 29-30.

[9] Пат. РФ № 2404087. Термоэмиссионный способ тепловой защиты частей летательных аппаратов при их аэродинамическом нагреве. / В.А. Керножицкий, А.В. Колычев, Д.М. Охочинский. 2010 Бюл. № 32.7 с.

[10] Пат. РФ № 2430857. Крыло высокоскоростного звукового летательного аппарата в условиях его аэродинамического нагрева. / В.А. Керножицкий, А.В. Колычев, Д.М. Охочинский. 2011. Бюл. № 38.9 с.

[11] Пат. На полезную модель РФ № 95637. Крыло высокоскоростного звукового летательного аппарата в условиях его аэродинамического нагрева. / В.А. Керножицкий, А.В. Колычев, Д.М. Охочинский. 2010. Бюл. № 19.12 с.

[12] Пат. РФ № 2495788. Крыло гиперзвукового летательного аппарата в условиях его аэродинамического нагрева. / В.А. Керножицкий, В.Д. Атамасов. 2013. Бюл. № 29. 8 с.

[13] Пат. РФ № 2506199. Крыло гиперзвукового летательного аппарата в условиях его аэродинамического нагрева. / В.А. Керножицкий, А.В. Колычев, Д.М. Охочински. 2014. Бюл. № $4.11 \mathrm{c}$.

[14] Пат. РФ № 2572009. Крыло гиперзвукового летательного аппарата в условиях его аэродинамического нагрева. / В.А. Керножицкий, А.В. Колычев. 2015. Бюл. № 36.11 с.

[15] Колычев А.В., Керножицкий В.А. // Электронный журнал „Труды МАИ“. 2012. Вып. 51. С. 1-18.

[16] Колычев А.В. // Электронный журнал „Труды МАИ“. 2013. Вып. 68. С. 1-15.

[17] Колычев А.В. // Электронный журнал „Труды МАИ“. 2014. Вып. 74. С. 1-28.

[18] Колычев А.В. // Электронный журнал „Труды МАИ““. 2014. Вып. 75. С. 1-23.

[19] Alkandry H., Hanquist K.M., Boyd I.D. // 11th AIAA/ASME Joint Thermophysics and Heat Transfer Conference. 16-20 June 2014, Atlanta, GA. AIAA Paper 2014-2674.

[20] Hanquist K.M., Boyd I.D. // 45th AIAA Thermophysics Conference 22-26 June 2015, Dallas, TX. AIAA Paper 2015235.

[21] Hanquist K.M., Haray K., Boyd I.D. // 46th AIAA Thermophysics Conference, 13-17 June 2016, Washington, D.C. AIAA Paper 2016-4433.

[22] Hanquist K.M., Haray K., Boyd I.D. // J. Thermophysics and heat Transfer. 2017. Vol. 31. N 2. P. 283-293.

[23] Hanquist K.M., Boyd I.D. // 55th AIAA Aerospace Sciences Meeting, 9-13 January 2017, Gaylord Texan, Grapevine, Texas, USA. AIAA Paper 2017-0900.

[24] Walker S.H., Rodgers F. // AIAA/CIRA 13th International Space Planes and Hypersonics Systems and Technologies. Capua, CE, Italy, 16-20 May 2005. AIAA Paper 2005-3253.

[25] Fay J.A., Riddell F.R. // J. Aeronautics Sciences. 1958. Vol. 25. P. $73-85$.

[26] Дубинкер Ю.Б., Донской А.А. Эластомерные теплозащитные материалы: Обзор иностранной технической литературы за 1960-1968 гг. М.: ОНТИ, 1969.

[27] Voland R.T., Huebner L.D., McClinton C.R. // International Astronautical Federation - 56th International Astronautical Congress. 2005. Fukuoka; Japan; 17-21 October 2005. Vol. 8. P. 5333-5342. 
[28] Glass D.E. // 17th AIAA International Space Planes and Hypersonic Systems and Technologies Conference. 2011, San Francisco, CA, USA, 11-14 April 2011. AIAA Paper 20112304.

[29] Uribarri L., Allen E.H. // 20th AIAA International Space Planes and Hypersonic Systems and Technologies Conference, 2015, Glasgow, UK, 6-9 July 2015. AIAA 2015-3674.

[30] Takamura S., Ohno N., Ye M.Y., Kuwabara T. // Contributions to Plasma Physics. 2004. Vol. 44. P. 126-137.

[31] Hanquist K.M., Boyd I.D. // 54th AIAA Aerospace Sciences Meeting, 2016, San Diego, USA, 4-8 January 2016. AIAA P. 2016-0507.

[32] Sheehan J.P., Hershkowitz N. // Plasma Sourc. Sci. Technol. 2011. Vol. 20. N 6. P. 1-22.

[33] Барышников А.С., Басаргин И.В., Бобашев С.В., Монахов Н.А., Попов П.А., Сахаров В.А., Чистякова М.В. // Письма в ЖТФ. 2015. Т. 41. Вып. 15. С. 83-89.

[34] Барышников А.С., Басаргин И.В., Бобашев С.В., Монахов Н.А., Попов П.А., Сахаров В.А., Чистякова М.В. // Письма в ЖТФ. 2017. Т. 43. Вып. 22. С. 26-31.

[35] Hara K. Ph. D. Dissertation, Department of Aerospace Engineering, University of Michigan, Ann Arbor, MI, 2015.

[36] Hanquist K.M., Hara K., Boyd I.D. // J. Appl. Phys. 2017. Vol. 121. P. 053302-1-053302-14.

[37] Пат. РФ № 2573551. Способ охлаждения лопаток турбин газотурбинных установок. / А.В. Колычев, В.А. Керножицкий. 2016. Бюл. № 2.7 с.

[38] Пат. РФ № 2578387. Устройство охлаждения лопаток турбин газотурбинных установок. / А.В. Колычев, В.А. Керножицкий. 2016. Бюл. № 9.8 с.

[39] Пат. РФ № 151082. Устройство охлаждения лопаток турбины газотурбинной установки. / А.В. Колычев, В.А. Керножицкий, М.Н. Охочинский 2015. Бюл. № 8.8 с.

[40] Haibo Gan // 30th International Vacuum Nanoelectronics Conference (IVNC), 10-14 July 2017, Regensburg, Germany. P. 116-117.

[41] Rastegar I. et al. // 30th International Vacuum Nanoelectronics Conference (IVNC), 10-14 July 2017, Regensburg, Germany. P. 54-55.

[42] Ruei-Fu Jao et al. // 30th International Vacuum Nanoelectronics Conference (IVNC), 10-14 July 2017, Regensburg, Germany. P. 26-27.

[43] Yoshizumi T., Hayashi K. // Appl. Phys. Express. 2013. Vol. 6. N 1. P. 015802-1-015802-3.

[44] Масленников В.Г., Сахаров В.А. // ЖТФ. 1997. Т. 67. Вып. 11. С. 88-95. 